\title{
Effets saisonniers sur les relations poids-longueurs et coefficients de condition pour 16 espèces de poissons de la Lagune Mvassa, basse Guinée, République du Congo
}

Hélène Dembe Louvinguila Tenda ${ }^{1,2}$, Armel Ibala Zamba ${ }^{1,2}$, Joseph Goma-Tchimbakala ${ }^{1,2}$, Lérège Batiabo Mikembi ${ }^{2}$, Honest Freedom Poaty Ngot $^{2}$, Victor Mamonekene ${ }^{1,2}$

${ }^{1}$ Ecole Nationale Supérieure d'Agronomie et de Foresterie (ENSAF), Université Marien Ngouabi, Brazzaville, République du Congo

2Institut national de Recherche en Sciences Exactes Naturelles, Laboratoire de l'Environnement et Océanographie, Brazzaville, République du Congo.

*Auteur correspondant : Email : ibalaszamba@yaboo.fr / Tél : +242066739574

Mots clés : Poissons, croissance, coefficient de condition, saison, lagune Mvassa

Publication date 30/04/2020, http://m.elewa.org/Joutnals/about-japs/

1 RÉSUMÉ

Objectifs: La présente étude a été initiée pour établir les relations poids-longueur et les facteurs de condition de Ethmalosa fimbriata, Sardinella maderensis, Coptodon guinensis, Oreochromis schwebischi, Neochelon falcipinnis, Mugil cephalus, Parachelon grandisquamis, Eucinostomus melanopterus, Hemichromis elongatus, Pomadasys jubelini, Eleotris daganensis, Bathygobius soporator, Trachinotus teraia, Lutjanus goreensis, Mugil banannensis, et Sarotherodon melanotheron, 16 espèces les plus abondantes de la lagune Mvassa en République du Congo.

Méthodologie et résultats: Les échantillonnages des poissons ont été réalisés sur 9 stations à l'aide des filets maillants de $8,10,20$ et $30 \mathrm{~mm}$ de côté de la maille. Les relations poidslongueur ont été réalisées en utilisant l'équation $\mathbf{P}=a \mathbf{L S}{ }^{b}$ et leurs coefficients de condition en utilisant l'équation $\mathrm{K}=100 *\left(\mathrm{P} / \mathrm{LS}^{b}\right)$. Au total, 1566 spécimens, réparties en 5 ordres, 8 familles, 16 espèces dont 10 ont été capturées en saison sèche, 14 en saison des pluies et 8 espèces communes pour les deux saisons ont été utilisés dans le cadre de cette étude. Le coefficient de détermination $r^{2}$ était significatif pour les deux saisons et variait de 0,91 pour Neochelon falcipinnis et 0,99 pour Pomadasys jubelini avec une moyenne de 0,97 $\pm 0,02$. En saison sèche, le coefficient d'allométrie $b$ de la relation poids-longueur variait de 2,04 pour Mugil cephalus à 3,48 Sardinella maderensis avec une moyenne de 2,79 $\pm 0,03$. Le facteur de condition variait en moyenne de 0,66 \pm 0,06 pour Sardinella maderensis à 12,22 \pm 0,28 pour Mugil cephalus. En saison des pluies, le coefficient d'allometrie $b$ variait de 2,41 pour Eucinostomus melanopterus à 3,94 pour Sardinella maderensis, avec une moyenne de 3,01 \pm 0,05. Le facteur de condition variait de 0,02 \pm 0,002 pour Parachelon grandisquamis à 6,73 \pm 0,41 pour Eucinostomus malanopterus. Pour les huit espèces communes, le coefficient d'allometrie $b$ était plus élevé en saison de pluie qu'en saison sèche pour la plupart des espèces $(63 \%)$.

Conclusion et application des résultats : Ces résultats rapportent des informations relatives à la biologie des poissons rencontrés dans la lagune Mvassa, mais aussi sur l'influence de la saison sur les relations poids-longueur et les coefficients de condition. 
Seasonal effects on weight-length relationships and condition factors for 16 fish species from the Mvassa Lagoon, Lower Guinea, Republic of the Congo

\begin{abstract}
Objectives: This study was initiated to establish weight-length relationships and condition factors of Ethmalosa fimbriata, Sardinella maderensis, Coptodon guinensis, Oreochromis schwebischi, Neochelon falcipinnis, Mugil cephalus, Parachelon grandisquamis, Eucinostomus melanopterus, Hemichromis elongatus, Pomadasys jubelini, Eleotris daganensis, Bathygobius soporator, Trachinotus teraia, Lutjanus goreensis, Mugil banannensis, et Sarotherodon melanotheron, 16 most abundant species of the Mvassa lagoon in the Republic of Congo.

Methodology and results: Fish samples were taken at 9 stations utilizing 8, 10, 20 and $30 \mathrm{~mm}$ mesh gillnets. The weight-length relationships were performed using equation $\mathbf{P}=\mathrm{aLS}^{\mathrm{b}}$ and their condition coefficients using equation $\mathrm{K}=100 *\left(\mathrm{P} / \mathrm{LS}^{b}\right)$. A total of 1566 specimens, divided into 5 orders, 8 families, 16 species of which 10 were caught in the dry season, 14 in the rainy season and 8 common species for both seasons were used in this study. The correlation coefficient $r^{2}$ was significant for both seasons and ranged from 0.91 for Neochelon falcipinnis and 0.99 for Pomadasys jubelini with an average of $0.97 \pm 0.02$. In the dry season, the allometry coefficient $b$ of the weight-length relationship ranged from 2.04 for Mugil cephalus to 3.48 Sardinella maderensis with an average of $2.79 \pm 0.03$. The condition factor ranged on average from $0.66 \pm 0.06$ for Sardinella maderensis to $12.22 \pm 0.28$ for Mugil cephalus. In the rainy season, the allometry coefficient b ranged from 2.41 for Eucinostomus melanopterus to 3.940 for Sardinella maderensis, with an average of $3.014 \pm 0.05$. The condition factor ranged from $0.02 \pm 0.002$ for Parachelon grandisquamis to $6.73 \pm 0.41$ for Eucinostomus malanopterus. For the eight common species, the allometry coefficient $b$ was higher in the rainy season than in the dry season for most species $(63 \%)$.

Conclusion and application of the results: These results report information on the biology of the fish encountered in the Mvassa lagoon, but also on the influence of the season on weight- length relationships and condition coefficients
\end{abstract}

\section{INTRODUCTION}

Les écosystèmes aquatiques, bien que représentant un faible pourcentage de la surface de la Terre, remplissent divers rôles et fonctions, dont l'humanité bénéficie (RIOB, 2015). Ces écosystèmes constituent dès lors des ressources indispensables à la vie (Konan et al., 2017). Malgré leur important rôle, ces milieux subissent plusieurs pressions d'origine naturelle et anthropiques, entrainant ainsi leur dégradation et la perte de la biodiversité (Lévêque et al., 1990 ; Mostarih et al., 2016). Les eaux africaines en générale et celles de la base Guinée en particulier ne sont pas épargnées par cette situation, alors qu'elles renferment une forte diversité biologique. La protection de ces milieux ne peut être efficace sans une bonne connaissance systématique, biologique et écologique des êtres qui y vivent (Lalèyé, 2006). La relation poids-longueur (RLP) est un outil important en biologie, physiologie, écologie et évaluation des stocks pour la gestion et la conservation des populations naturelles des poissons (Écoutin et Albaret, 2003 ; Hossain et al., 2006, Hossain et al., 2012 ; Bolognini et al., 2013). En effet, elle permet de prédire le poids d'un poisson, connaissant sa longueur, lors de l'appréciation des rendements des pêches (Écoutin et Albaret, 2003; Froese, 2006 ; Froese et al., 2014). Le coefficient de condition fournit des informations sur l'état d'embonpoint d'un poisson (Lévêque et al., 1990). Il constitue un instrument souvent utilisé 
pour comparer l'état physiologique global de populations au cours d'un cycle saisonnier ou entre bassins présentant des conditions écologiques similaires ou différentes (Le Cren, 1951). Fortement influencé par les paramètres de l'environnement, le coefficient de condition peut être utilisé comme un indice pour évaluer le niveau de perturbation d'un écosystème aquatique (Baby, 2011). L'histoire ichtyologique des bassins côtiers de la République du Congo est assez récente et peu étoffée. Les études biologiques, physiologiques et écologiques basées sur les relations poids-longueur et coefficients de condition des poissons de la région ichtyogégraphique de basse Guinée en général ont jusque-là rarement été entreprises.

\section{MATERIEL E METHODES}

\subsection{Présentation du milieu et stations} échantillonnées : La présente étude a été conduite dans la lagune Mvassa, située dans le Département de Pointe-Noire, au sud-ouest de la République du Congo (figure 1). Ce plan d'eau s'étend sur environ 12 hectares, elle est alimentée par trois petits ruisseaux en amont et se communique de manière temporaire avec l'Océan. Elle s'ouvre naturellement en saison de pluie et reste fermée en saison sèche. Les poissons ont été échantillonnés dans trois stations, choisies en fonction de la distance avec l'embouchure, incluant chacune trois biotopes: Mangrove, Herbe et Pleine eau (Figure 1). Huit campagnes de récoltes des données ont été réalisées entre 2018 et 2019, dont quatre en saison des pluies entre Janvier et Avril et quatre en saison sèche entre Juillet et Août. Les pêches ont été réalisées suivant une
La lagune Mvassa, l'un des écosystèmes d'eaux saumâtres de cette région, entièrement situé dans la ville de Pointe-Noire (République du Congo), est à ce jour vulnérable, du fait des pressions anthropiques chaque jour croissantes. Face à cette situation, il est important d'établir des mesures de conservation des populations des poissons de cette lagune. La présente étude a été initiée pour contribuer à la connaissance biologique de la faune ichtyologique de la lagune Mvassa en se basant sur les relations poids-longueur et les coefficients de condition des espèces les plus abondantes. L'étude fournie précisément les données des effets saisonniers sur la croissance et l'état d'embonpoint de 16 espèces de poissons.

méthode standardisée à l'aide des filets maillants de 8,10,20, $30 \mathrm{~mm}$ de côté de la maille. Seules les pêches de nuit ont été réalisées suivant Ibala Zamba et al. (2019). Les filets ont été posés le soir vers $16 \mathrm{~h}$ pour être retirés le lendemain matin vers $6 \mathrm{~h}$. Tous les spécimens de poissons capturés ont été identifiés sur le terrain. Ils ont été comptés par espèces, mesurés à 0,01 millimètre près de la Longueur Standard et pesés à $0,01 \mathrm{~g}$ près à l'aide d'une balance de précision. Les poissons dont les identifications ont été incertaines sur le terrain ont été préservés dans le formol à $10 \%$ pour les identifications au laboratoire ichtyologique de l'Institut National de Recherche en Sciences Exactes et Naturelles (IRSEN). La classification des familles a été faite suivant Van Der Laan et al., (2019) alors que les genres et les espèces ont été classés suivant l'ordre alphabétique. 


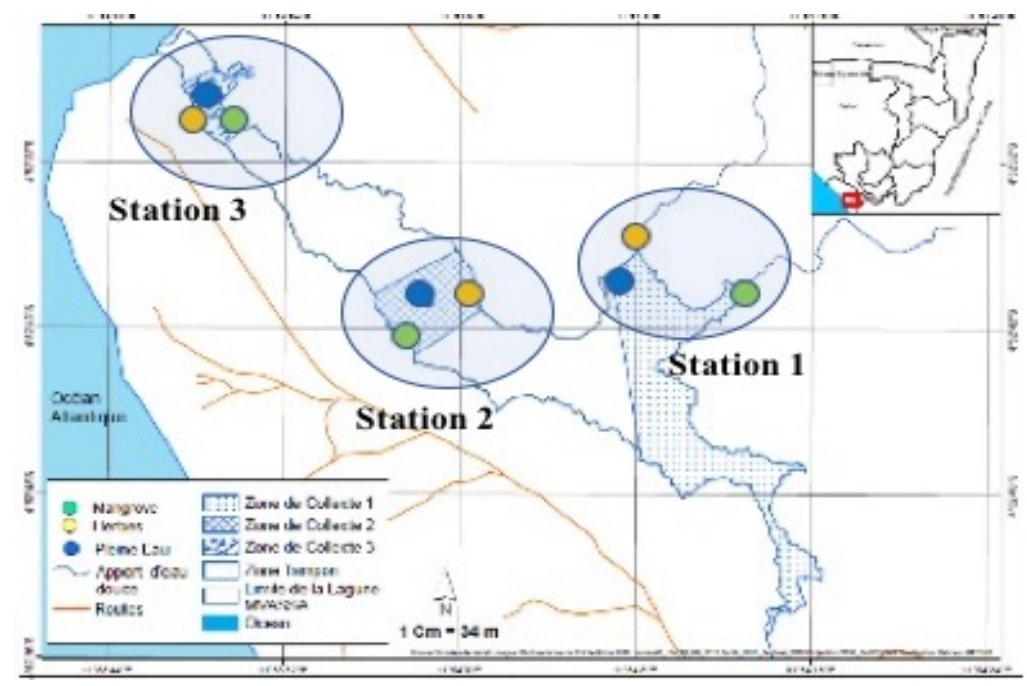

Figure 1 : Lagune Mvassa et les stations échantillonnées

3.2 Analyse des données: Les relations poids-longueur chez les poissons sont considérées comme des modèles de croissance allométrique (Palomares et al., 1966). Les courbes de régression du type puissance de formule $\mathrm{P}=a \mathrm{LS}^{b}$ ont été obtenues à partir des couples poids-longueurs, où $P$ représente le poids de l'individu (g), LS la longueur standard $(\mathrm{cm}), a$ le coefficient de croissance initial et $b$ la pente de la droite de régression. L'estimation des paramètres d'un tel modèle est réalisée par régression linéaire sur les données ayant subi une transformation logarithmique $(\log \mathrm{P}=$ $\log a+b \operatorname{LogLS})$. Les paramètres $a$ et $b$ pour chacune des équations de la relation LongueurPoids ont été estimés par les analyses des régressions linéaires (Zar, 1999). La relation poids-longueur reflète une croissance isométrique (I) lorsque $b=3$ et une croissance allométrique lorsque $b \neq 3$. Cependant, une croissance allométrique positive (AP) est observée lorsque $b>3$ et une est une croissance allométrique négative (AN) lorsque $b$ $<3$ (Shingleton, 2010). L'intervalle de

\section{RESULTATS}

Un total de 1566 spécimens répartis en 5 ordres, 9 familles et 16 espèces ont été rapportées dans le cadre de la présente étude. Parmi les familles rapportées, les Mugilidae sont les plus abondants $(41,25 \%)$ suivis des confiance de $a$ et de $b$ à $95 \%$ a été estimé à l'aide du logiciel Statview version 1992-98 (SAS Institute INC). La différence statistique entre la valeur de $b$ pour chacune des espèces et la valeur isométrique $(b=3)$ a été obtenue à l'aide du test de $t$ de Student réalisé suivant Sokal et Rohlf $(1987):$ ts $=(b-3) / \mathrm{ES} b$, où ts est la valeur de $t$ du test de $t$ de Student, $b$ la pente de la droite de régression et ESb l'erreur standard de $b$. Tous les tests ont été significatifs au seul de $5 \%(p<0,05)$. Pour évaluer l'état d'embonpoint des poissons de la lagune Mvassa, la valeur individuelle du facteur de condition pour chacun des spécimens a été calculée en utilisant la formule $\mathrm{K}=\left(100 \mathrm{P} / \mathrm{LS}^{\natural}\right)$ (Bagenal et Tesch, 1978), où $\mathrm{P}$ représente le poids de l'individu ( $\mathrm{g}$ ) et LS la longueur standard $(\mathrm{cm})$. Dans le cadre de la présente étude, seules les espèces représentées au moins par 10 spécimens ont été retenues, comme suggéré par Lalèyè (2006) Konan et al. (2017), Lederoun et al. (2016), Tah et al. (2016), Batiabo et al. 2(019) et Ibala Zamba et al. (2020).

Cichlidae (36,9\%), des Clupeidae (9,32\%), des Gerreidae (6,83\%), des Lutjanidae (3,32\%), des Gobiidae $(0,89)$, des Eleotridae $(0,70)$, des Carangidae et des Haemulidae $(0,63 \%)$ chacune. En considérant les saisons, 1116 spécimens 
appartenant à 5 ordres, 8 familles et 14 espèces ont été rapportées en saison des pluies et 450 spécimens appartenant à 5 ordres, 5 familles et 10 espèces ont été rapportées en saison sèche (tableau 1). En saison des pluies la famille des Cichlidae $(44 \%)$ était la plus abondante suivie de celles des Mugilidae (34\%), des Clupeidae $(9 \%)$, des Gerreidae et Lutjanidae avec chacune $(5 \%)$ et des Eleotridae, Gobiidae et Carangidea avec chacune (1\%). En saison sèche la famille des Mugilidae (58\%) était la plus abondante suivie celle des Cichlidae (18\%), des Gerreidae (12), Clupeidae $(10 \%)$ et des Haemulidae (2\%). Sur les 16 espèces retenues, huit (8) espèces ont été capturées aussi bien en saison de sèche qu'en saison des pluies (Ethmalosa fimbriata, Sardinella maderensis, Coptodon guinensis, Oreochromis schwebischi, Neochelon falcipinnis, Mugil cephalus, Parachelon grandisquamis et Eucinostomus melanopterus). Deux espèces ont été rapportées uniquement en saison sèche (Hemichromis elongatus et Pomadasys jubelini) et 6 uniquement en saison de pluie (Eleotris daganensis, Bathygobius soporator, Trachinotus teraia, Lutjanus goreensis, Mugil banannensis, et Sarotherodon melanotheron). La taille de l'échantillon variait de 11 spécimens pour Trachinotus teraia à 358 spécimens pour Oreochromis schwebischi en saison des pluies et de 10 spécimens pour Pomadasys jubelini à 236 pour Neochelon falcipinnis en saison sèche. Pour les deux saisons le coefficient de corrélation $\left(r^{2}\right)$ était supérieur à 0,90 . Les valeurs de $r^{2}$ varient entre 0,918 pour Neochelon falcipinnis et 0,99 pour Pomadasys jubelini. Le coefficient d'allometrie $b$ variait de 2,04 pour Mugil cephalus à 3,48 Sardinella maderensis en saison sèche et de 2,41 pour Eucinostomus melanopterus à 3,94 pour Sardinella maderensis en saison des pluies. Les types de croissance sont consignés dans le tableau 1. En saison des pluies six espèces (Oreochromis schwebischi, Eucinostomus melanopterus,
Coptodon guinensis, Parachelon gandisquamis, Sarotherodon melanotheron et Mugil banaensis) ont présenté une croissance allométrique négative $(b$ $<3 ; \mathrm{p}<0,05$ ), quatre (Eleotris daganensis, Sardinella maderensi, Trachinotus teraia et Ethmalosa fimbriata) ont une croissance allométrique positive $(b>3 ; \mathrm{p}<0,05)$ et quatre (Parachelon grandisquamis, Mugil cephalus et Bathygobius soporator et Lutjanus goreensis) ont une croissance isométrique $(b=3 ; \mathrm{p}>0,05)$. En saison sèche cinq espèces (Ethmalosa fimbriata, Oreochromis schwebischi, Parachelon grandisquamis, Pomadasys jubelini et Eucinostomus melanopterus) ont montré une croissance isométrique $(b=3 ; \mathrm{p}>0,05)$, quatre espèces (Coptodon guinenensi, Hemichromis elongatus, Mugil cephalus et Neochelon falcipinnis) ont montré une croissance allométrique négative ( $b$ $<3 ; \mathrm{p}<0,05$ ) et une espèce (Sardinella maderensis) a montré croissance allométrique positive $(b>3 ; \mathrm{p}<0,05)$. Les résultats sur les valeurs moyennes et extrêmes de paramètres étudiés (Poids et Longueur) et les coefficients de condition $\mathrm{K}$ sont consignés dans le tableau 2. L'examen du tableau 2 montre qu'en saison des pluies, la longueur la plus faible a été observée chez Oreochromis schwebischi $(3,55 \mathrm{~cm})$ et la plus élevée chez Parachelon grandisquamis $(15,75 \mathrm{~cm})$ mais le poids le plus faible a été observé chez Ethmalosa fimbriata $(0,82 \mathrm{~g})$ et le plus élevé chez Lutjanus goreensis $(86,5 \mathrm{~g})$. Les coefficients de condition $\mathrm{K}$ variaient de $0,02 \pm$ 0,002 pour Parachelon grandisquamis à $6,73 \pm 0,41$ pour Eucinostomus malanopterus. En saison sèche la longueur et poids les plus faibles ont été observés chez Ethmalosa fimbriata $(2,93 \mathrm{~cm}$ et $0,36 \mathrm{~g})$ et la plus élevée chez Neochelon falcipinnis $(14,83 \mathrm{~cm}$ et $50,1 \mathrm{~g})$. Les coefficients de condition variaient de $0,66 \pm 0,06$ pour Sardinella maderensis à 12,22 $\pm 0,28$ pour Mugil cephalus. 
Tableau 1 : Le nombre de spécimens et les paramètres estimés des relations poids-longueur pour les 16 espèces de poissons de la lagune Mvassa

\begin{tabular}{|c|c|c|c|c|c|c|c|c|c|}
\hline Famille et Espèces & saisons & $\mathbf{N}$ & $a$ & $\begin{array}{c}\text { IC de a à } 95 \\
\%\end{array}$ & $b$ & $\begin{array}{c}\text { IC de b à } \\
95 \%\end{array}$ & $\begin{array}{c}\text { ES de } \\
\text { b }\end{array}$ & $r^{2}$ & $\begin{array}{c}\text { Type de } \\
\text { croissance }\end{array}$ \\
\hline \multicolumn{10}{|l|}{ Clupeidae } \\
\hline Ethmalosa fimbriata (Bowdich, 1825) & SS & 31 & 0,016 & $0,013-0,021$ & 2,943 & $2,770-3,117$ & 0,085 & 0,976 & I \\
\hline Ethmalosa fimbriata (Bowdich, 1825) & SP & 89 & 0,016 & $0,013-0,019$ & 3,148 & $3,050-3,248$ & 0,050 & 0,978 & AP \\
\hline Sardinella maderensis (Lowe, 1838) & SS & 14 & 0,006 & $0,005-0,009$ & 3,488 & $3,334-3,644$ & 0,07 & 0,995 & $\mathrm{AP}$ \\
\hline Sardinella maderensis (Lowe, 1838) & SP & 12 & 0,009 & $0,002-0,003$ & 3,940 & $3,808-4,020$ & 0,048 & 0,991 & AP \\
\hline \multicolumn{10}{|l|}{ Eleotridae } \\
\hline Eleotris daganensis Steindachner, 1870 & SP & 11 & 0,017 & $0,015-0,020$ & 3,175 & $3,096-3,252$ & 0,034 & 0,995 & $\mathrm{AP}$ \\
\hline \multicolumn{10}{|l|}{ Gobiidae } \\
\hline Bathygobius soporator Valenciennes, 1837) & SP & 14 & 0,013 & $0,013-0,041$ & 2,843 & $2,531-3,155$ & 0,143 & 0,993 & $\mathrm{I}$ \\
\hline \multicolumn{10}{|l|}{ Cichlidae } \\
\hline Coptodon guineensis (Günther, 1862) & SS & 38 & 0,055 & $0,046-0,067$ & 2,783 & $2,656-2,844$ & 0,063 & 0,981 & $\mathrm{AN}$ \\
\hline Coptodon guineensis (Günther, 1862) & SP & 105 & 0,054 & $0,046-0,064$ & 2,740 & $2,637-2,844$ & 0,052 & 0,963 & $\mathrm{AN}$ \\
\hline Hemichromis elongatus (Guichenot, 1861) & SS & 29 & 0,095 & $0,082-0,146$ & 2,366 & $2,167-2,567$ & 0,097 & 0,956 & $\mathrm{AN}$ \\
\hline Oreochromis schwebischi (Sauvage, 1884) & SS & 12 & 0,047 & $0,030-0,076$ & 2,963 & $2,656-3,270$ & 0,013 & 0,996 & $\mathrm{I}$ \\
\hline Oreochromis schwebischi (Sauvage, 1884) & SP & 358 & 0,061 & $0,055-0,068$ & 2,741 & $2,669-2,814$ & 0,037 & 0,939 & $\mathrm{AN}$ \\
\hline Sarotherodon melanotheron Rüppell, 1852 & SP & 28 & 0,056 & $0,051-0,058$ & 2,867 & $2,846-2,913$ & 0,016 & 0,997 & $\mathrm{AN}$ \\
\hline \multicolumn{10}{|l|}{ Mugilidae } \\
\hline Mugil bananeensis (Pellegrin, 1928) & SP & 64 & 0,042 & $0,037-0,050$ & 2,688 & $2,614-2,764$ & 0,038 & 0,988 & $\mathrm{AN}$ \\
\hline Mugil cephalus Linnaeus, 1758 & SS & 14 & 0,135 & $0,118-0,015$ & 2,047 & $1,976-2,117$ & 0,032 & 0,997 & $\mathrm{AN}$ \\
\hline Mugil cephalus Linnaeus, 1758 & SP & 15 & 0,025 & $0,020-0,034$ & 2,995 & $2,863-3,128$ & 0,061 & 0,994 & $\mathrm{I}$ \\
\hline Neochelon falcipinnis (Valenciennes, 1836) & SS & 236 & 0,057 & $0,048-0,059$ & 2,499 & $2,403-2,595$ & 0,049 & 0,918 & $\mathrm{AN}$ \\
\hline Neochelon falcipinnis (Valenciennes, 1836) & SP & 254 & 0,028 & $0,025-0,034$ & 2,884 & $2,798-2,972$ & 0,044 & 0,944 & $\mathrm{AN}$ \\
\hline Parachelon grandisquamis (Valenciennes, 1836) & SS & 11 & 0,023 & $0,014-0,041$ & 2,974 & $2,704-3,240$ & 0,011 & 0,987 & $\mathrm{I}$ \\
\hline Parachelon grandisquamis (Valenciennes, 1836) & SP & 52 & 0,023 & $0,019-0,028$ & 2,979 & $2,885-3,075$ & 0,047 & 0,987 & I \\
\hline \multicolumn{10}{|l|}{ Carangidae } \\
\hline Trachinotus teraia Cuvier, 1832 & SP & 10 & 0,007 & $0,004-0,15$ & 3,661 & $3,221-4,103$ & 0,119 & 0,978 & AP \\
\hline \multicolumn{10}{|l|}{ Lutjanidae } \\
\hline Lutjanus goreensis (Valenciennes, 1830) & SP & 52 & 0,024 & $0,011-0,030$ & 3,106 & $3,005-3,206$ & 0,050 & 0,986 & I \\
\hline \multicolumn{10}{|l|}{ Gerreidae } \\
\hline Eucinostomus melanopterus (Bleeker, 1863) & SS & 55 & 0,026 & $0,020-0,036$ & 2,931 & $2,758-3,105$ & 0,086 & 0,955 & I \\
\hline Eucinostomus melanopterus (Bleeker, 1863) & SP & 52 & 0,066 & $0,048-0,091$ & 2,41 & $2,225-2,595$ & 0,033 & 0,932 & $\mathrm{AN}$ \\
\hline \multicolumn{10}{|l|}{ Haemulidae } \\
\hline Pomadasys jubelini (Cuvier, 1830) & SS & 10 & 0,029 & $0,025-0,034$ & 2,940 & $2,855-3,027$ & 0,037 & 0,998 & $\mathrm{I}$ \\
\hline
\end{tabular}

$a$ : coefficient de croissance ; $b$ : pente de la droite de régression ; IC : intervalle de confiance ; ES : erreur standard ; $r^{2}:$ coefficient de détermination. 
Tableau 2 : Descriptions statistiques obtenues pour les 16 espèces de poissons et leurs Coefficients de condition $(\mathrm{K})$

\begin{tabular}{|c|c|c|c|c|c|c|c|c|}
\hline \multirow[b]{2}{*}{ Famille et Espèces } & \multirow[b]{2}{*}{ Saisons } & \multirow[b]{2}{*}{$\mathbf{N}$} & \multicolumn{2}{|c|}{ Longueur (cm) } & \multicolumn{2}{|c|}{ Poids (g) } & \multicolumn{2}{|c|}{$\mathbf{K}$} \\
\hline & & & Min-Max & Moy \pm DS & Min-Max & Moy \pm DS & $\begin{array}{l}\text { Min- } \\
\text { Max }\end{array}$ & Moy \pm DS \\
\hline \multicolumn{9}{|l|}{ Clupeidae } \\
\hline Ethmalosa fimbriata (Bowdich, 1825) & SS & 31 & $2,93-9,8$ & $4,62 \pm 1,69$ & $0,36-13,39$ & $2,22 \pm 3,55$ & $1,17-2,15$ & $1,77 \pm 0,22$ \\
\hline Ethmalosa fimbriata (Bowdich, 1825) & SP & 89 & $3,61-11,74$ & $6,98 \pm 1,61$ & $0,82-36,94$ & $8,77 \pm 6,97$ & $1,44-2,75$ & $1,76 \pm 0,20$ \\
\hline Sardinella maderensis (Lowe, 1838) & SS & 14 & $4,35-12,1$ & $5, \pm 2,07$ & $1,1-41,96$ & $5,55 \pm 10,66$ & $0,50-0,82$ & $0,66 \pm 0,06$ \\
\hline Sardinella maderensis (Lowe, 1838) & SP & 12 & $4,79-6,53$ & $5,57 \pm 0,50$ & $1,34-4,56$ & $2,54 \pm 0,92$ & $0,29-0,33$ & $0,30 \pm 0,01$ \\
\hline \multicolumn{9}{|l|}{ Eleotridae } \\
\hline Eleotris daganensis Steindachner, 1870 & SP & 11 & $5,01-7,4$ & $5,812 \pm 0,71$ & $2,88-9,86$ & $4,79 \pm 2,06$ & $1,68-1,67$ & $1,63 \pm 0,02$ \\
\hline \multicolumn{9}{|l|}{ Gobiidae } \\
\hline $\begin{array}{l}\text { Bathygobius soporator Valenciennes, } \\
\text { 1837) }\end{array}$ & SP & 14 & 4,24-7,93 & $6,16 \pm 0,86$ & $1,28-8,4$ & $4,26 \pm 1,61$ & $2,15-2,68$ & $2,50 \pm 0,17$ \\
\hline \multicolumn{9}{|l|}{ Cichlidae } \\
\hline Coptodon guineensis (Günther, 1862) & SS & 38 & $3,09-9,39$ & $4,62 \pm 1,53$ & $1,42-29,18$ & $5,20 \pm 6,68$ & $4,41-6,6$ & $5,42 \pm 0,54$ \\
\hline Coptodon guineensis (Günther, 1862) & SP & 105 & $3,61-9,98$ & $5,03 \pm 1,74$ & $1,89-31,97$ & $6,16 \pm 7,63$ & $4,03-8,31$ & $5,87 \pm 0,85$ \\
\hline $\begin{array}{l}\text { Hemichromis elongatus (Guichenot, } \\
\text { 1861) }\end{array}$ & SS & 29 & $4,50-5,93$ & $5,05 \pm 0,32$ & $3,39-6,59$ & $4,46 \pm 0,71$ & $8,32-9,58$ & $9,08 \pm 0,28$ \\
\hline $\begin{array}{l}\text { Oreochromis schwebischi (Sauvage, } \\
\text { 1884) }\end{array}$ & SS & 12 & $3,72-5,23$ & $4,28 \pm 0,40$ & $2,23-5,84$ & $3,57 \pm 1,01$ & $4,80-5,56$ & $5,14 \pm 0,21$ \\
\hline $\begin{array}{l}\text { Oreochromis schwebischi (Sauvage, } \\
\text { 1884) }\end{array}$ & SP & 358 & $3,50-6,73$ & $4,51 \pm 0,46$ & $1,97-12,47$ & $3,94 \pm 1,27$ & $4,72-7,84$ & $6,53 \pm 0,44$ \\
\hline $\begin{array}{l}\text { Sarotherodon melanotheron Rüppell, } \\
1852\end{array}$ & SP & 28 & $5,37-9,02$ & $7,89 \pm 0,92$ & $6,85-30,39$ & $21,64 \pm 6,11$ & 6,16-6,91 & $6,42 \pm 0,09$ \\
\hline \multicolumn{9}{|l|}{ Mugilidae } \\
\hline Mugil bananeensis (Pellegrin, 1928) & SP & 64 & $5,88-7,5$ & $7 \pm 0,34$ & 4,99-9,75 & $8,08 \pm 1,05$ & $4,80-5,36$ & $5,09 \pm 0,09$ \\
\hline Mugil cephalus Linnaeus, 1758 & SS & 14 & $5,54-10,99$ & $6,86 \pm 1,51$ & $4,64-18,57$ & $7,32 \pm 3,79$ & $\begin{array}{l}11,70- \\
12,72\end{array}$ & $\begin{array}{c}12,22 \pm \\
0,28\end{array}$ \\
\hline Mugil cephalus Linnaeus, 1758 & SP & 15 & $5,62-12,02$ & $8,01 \pm 1,93$ & $4,48-45,3$ & $\begin{array}{c}15,44 \pm \\
11,99\end{array}$ & $2,76-3,37$ & $3,14 \pm 0,17$ \\
\hline $\begin{array}{l}\text { Neochelon falcipinnis (Valenciennes, } \\
\text { 1836) }\end{array}$ & SS & 236 & $3,71-14,83$ & $6,52 \pm 0,99$ & $1,7-50,1$ & $6,58 \pm 4,11$ & $4,36-7,65$ & $5,77 \pm 0,54$ \\
\hline $\begin{array}{l}\text { Neocbelon falcipinnis (Valenciennes, } \\
\text { 1836) }\end{array}$ & SP & 254 & $4,49-15,05$ & $6,65 \pm 1,62$ & $1,65-67,99$ & $8,09 \pm 10,33$ & $1,68-5,06$ & $2,77 \pm 0,36$ \\
\hline Parachelon grandisquamis & SS & 11 & $5,73-12,98$ & $7,43 \pm 1,92$ & $4,29-49,37$ & $11,38 \pm$ & $2,51-3,17$ & $2,79 \pm 0,21$ \\
\hline
\end{tabular}




\begin{tabular}{|c|c|c|c|c|c|c|c|c|}
\hline $\begin{array}{l}\text { (Valenciennes, 1836) } \\
\text { Parachelon grandisquamis } \\
\text { (Valenciennes, 1836) }\end{array}$ & SP & 52 & $4,61-15,75$ & $8,40 \pm 3,01$ & $1,91-83,9$ & $\begin{array}{c}12,72 \\
18,85 \pm \\
23,62\end{array}$ & $0,02-0,03$ & $\begin{array}{l}0,02 \pm \\
0,002\end{array}$ \\
\hline \multicolumn{9}{|l|}{ Carangidae } \\
\hline Trachinotus teraia Cuvier, 1832 & SP & 10 & $4,15-8,31$ & $5,03 \pm 1,22$ & $1,08-17,21$ & $3,66 \pm 4,81$ & $0,55-0,85$ & $0,69 \pm 0,07$ \\
\hline \multicolumn{9}{|l|}{ Lutjanidae } \\
\hline $\begin{array}{l}\text { Lutjanus goreensis (Valenciennes, } \\
1830 \text { ) }\end{array}$ & SP & 52 & $3,84-13,65$ & $6,41 \pm 2,35$ & $1,8-86,5$ & $\begin{array}{c}12,36 \pm \\
17,70\end{array}$ & $1,81-3,14$ & $2,45 \pm 0,26$ \\
\hline \multicolumn{9}{|l|}{ Gerreidae } \\
\hline $\begin{array}{l}\text { Eucinostomus melanopterus (Bleeker, } \\
\text { 1863) }\end{array}$ & SP & 52 & 4,62-6,98 & $5,68 \pm 0,57$ & $2,35-7,19$ & $4,43 \pm 1,09$ & $5,65-7,26$ & $6,73 \pm 0,41$ \\
\hline $\begin{array}{l}\text { Eucinostomus melanopterus (Bleeker, } \\
\text { 1863) }\end{array}$ & SS & 31 & $2,93-9,8$ & $4,62 \pm 1,69$ & $0,36-13,39$ & $2,22 \pm 3,55$ & $1,17-2,15$ & $1,77 \pm 0,22$ \\
\hline \multicolumn{9}{|l|}{ Haemulidae } \\
\hline Pomadasys jubelini (Cuvier, 1830) & SS & 10 & $5,03-6,62$ & $5,62 \pm 0,56$ & $3,32-7,59$ & $4,81 \pm 1,48$ & $3,05-3,18$ & $3,13 \pm 0,03$ \\
\hline
\end{tabular}

Min : minimum ; Max : maximum ; Moy : moyenne ; DS : déviation standard ; K : coefficient de condition. 


\section{DISCUSSION}

L'analyse des RPLs montre que le poids est fortement corrélé à la longueur, toutes les corrélations obtenues ont été significatives $\mathrm{p}<$ 0,05 avec les coefficients de détermination $r^{2}>$ 0,95 , sauf pour Neochelon falcipinnis $\left(r^{2}=0,91 \mathrm{en}\right.$ saison sèche et 0,94 en saison de pluie) et Oreochromis schwebischi $\left(r^{2}=0,93\right)$ en saison de pluie, qui pourrait être due à la taille de leur l'échantillon. Les valeurs positives et élevées des coefficients de détermination avec une moyenne de $r^{2}=0,976 \pm 0,023$ montrent que pour l'ensemble des espèces, la croissance en taille induit une augmentation de poids chez les poissons de la lagune Mvassa. Écoutin et Albaret (2003) ont obtenu les mêmes résultats pour les poissons lagunaires de l'Afrique de l'Ouest de même que Batiabo et al. (2019) pour les poissons de la rivière Dzoumouna au Congo. L'utilisation des filets de différentes tailles de mailles (8, 10, 20, $30 \mathrm{~mm}$ de côté) nous a permis d'avoir une gamme de variation en taille $(2,93-15,75 \mathrm{~cm})$ et en poids $(0,36-86,5$ $\mathrm{g}$ ), incluant ainsi les juvéniles et les adultes des espèces de poissons. Pour une bonne utilisation des relations poids-longueur, toutes les phases écologiques des poissons (les juvéniles et les adultes) doivent être prises en compte (Romdhani et al., 2010 ; Écoutin et Albaret, 2003 ; Froese, 2006 ; Petrakis et Lévêque, 2017). Faisant partie des espèces qui forment le peuplement lagunaire fondamental (Albaret, 1994), Oreochromis schwebischi et Neochelon falcipinnis sont les plus représentées. Le gonflement de la classe des juvéniles pour les deux espèces nous renseigne aussi sur le degré d'exploitation des ressources de cette lagune. Environ $90 \%$ des individus avaient une taille inférieure à $5 \mathrm{~cm}$ pour Oreochromis schwebischi et à $10 \mathrm{~cm}$ pour Neochelon falcipinnis. La forte gamme des juvéniles chez les deux espèces prouve que ces eaux sont fortement perturbées. Dans l'ensemble de l'étude, sans tenir compte du facteur saison, les poissons de la lagune Mvassa ont présenté un coefficient d'allométrie $b$ moyen de 2,92 $\pm 0,40$. Cette valeur n'est pas significativement différente de $3(p=0,10)$. La loi cubique de Froese (2006) pourrait être appliquée chez les espèces de la lagune Mvassa. Ces résultats sont conformes avec ceux obtenus par (Écoutin et Albaret, 2003) ces auteurs ont présenté un $b$ moyen de $2,96 \pm 0,20$ pour les poissons lagunaires de l'Afrique de l'Ouest. En tenant compte du facteur saison, on constate qu'en saison des pluies la pente de la droite de régression $b$ était de 3,01 $\pm 0,39$, cette valeur n'est pas significativement différente de 3 ( $\mathrm{p}=$ $0,58)$ contre $2,79 \pm 0,03$, significativement différente de $3(p=0,02)$. La loi cubique de Froese (2006) pourrait être appliquée chez les espèces de la lagune Mvassa en saison des pluies avec $b$ qui n'est pas significativement différent de 3 . Toutes les valeurs de $b$ obtenues pour les deux saisons sont conformes aux valeurs typiques de 2,5 à 3,5 (Froese, 2006, Carlander, 1969). Les espèces Hemichromis elongatus, Mugil cephalus, Neochelon falcipinnis en saison sèche et Eucinostomus melanopterus en saison des pluies ont présenté un coefficient d'allométrie $b$ inférieur à 2,5. Mais leurs valeurs se situent entre (2-4) habituellement trouvées chez les poissons d'eau douce (Bagenal et Tesch, 1978). Les poissons de lagune Mvassa ont présenté en saison sèche à $50 \%$ une croissance isométrique, le poisson croît proportionnellement en taille et en poids. Mais en saison des pluies $43 \%$ des espèces ont présenté une croissance allométrique négative, le poisson croit plus en taille au détriment du poids. Les espèces capturées uniquement en saison sèche ont présenté une croissance allométrique négative pour Hemichromis elongatus et isométrique pour Pomadasys jubelini. Les espèces capturées uniquement en saison des pluies ont présenté une croissance allométrique positive, le poids croît au détriment de la longueur, à l'exception de Lutjanus goreensis qui a une croissance isométrique. Ce sont des poissons marins Sardinella maderensis, Lutjanus gorensis, Trachinostus teraia, Eleotris daganensis, Bathygobius spoporator qui se sont introduites lors des ouvertures de la lagune. Ces espèces fréquentent la lagune pendant une période 
donnée et ne subissent pas assez de pression comme les espèces lagunaires strictes. Les espèces estuarienne avec affinité marine Mugil cephalus, Mugil bananensis, Neochelon falcipinnis, Parachelon grandisquamis ont présenté une croissance soit allométrique négative soit isométrique à l'exception de Ethmalosa fimbriata qui a présenté une croissance allométrique positive en saison des pluies. Toutes les espèces lagunaires stricts et les espèces lagunaires avec affinité continentale ont présenté une croissance allométrique négative en saison des pluies et allométrique négative ou isométrique en saison sèche (Coptpodon guinensis, Eucinostomus melanopterus et Sarotherodon mélanotheron, Hemichromis elongatus et Oreochromis schwebischi). Pour les huit espèces commune quatre ont présenté le même type de croissance pour les deux saisons Sardinella maderensis (AP), Coptodon guinensis (AN), Neochelon falcipinnis (AN) et Parachelon grandisquamis (I), quatre espèces ont présenté une situation différente, le type de croissance diffère d'une saison à l'autre. Oreochromis schwebischi et Eucinostomus melanopterus ont présenté une croissance isométrique en saison sèche et allométrique négative en saison des pluies, Ethmalosa fimbriata a présenté une croissance isométrique en saison sèche et allométrique positive en saison des pluies, Mugil cephalus a présenté une croissance allométrique négative en saison sèche et isométrique en saison des pluies. Le coefficient d'allometrie était supérieur en saison des pluies pour la plupart des espèces $(63 \%)$. Ces valeurs élevées en saison des pluies pourraient s'expliquer par le fait qu'en saison des pluies avant toute perturbation la nourriture est plus abondante et donc les poissons augmentent de poids en cette

\section{CONCLUSION}

Ce travail avait pour objectif de montrer l'influence de la saison sur la relation poidslongueur et les coefficients de condition. $\mathrm{La}$ variation saisonnière a une influence sur certaines espèces, d'autres par contre ne sont pas affectées par cette variation. D'une manière générale, la loi cubique peut être appliquée chez période. Mais pour les espèces dont le $b$ est supérieur en saison sèche (Coptpodon guinensis et Eucinostomus melanopsterus) pourrait aussi être la conséquence des perturbations que cette lagune subit en saison des pluies. Les fortes précipitations entrainent ouverture de la lagune et l'eau se vide. Ces perturbations pourraient causer les stress aux poissons qui ne vivent strictement que dans ces milieux. Ces poissons subissent moins de migration sauf pour Oreochromis schsebischi qui est un poisson lagunaire à affinité continentale (Albaret, 1994). D’après Bagenal et Tesch (1978), les valeurs du coefficient de condition $(\mathrm{K})$ comprises entre 2,9 et 4,8 sont conformes pour les poissons d'eau douce. Dans le cadre de la présente étude, seuls Mugil cephalus $(3,14 \pm 0,17)$ en saison des pluies et Pomadasys jubelini $(3,13 \pm 0,03)$ en saison sèche, ont présenté des coefficients de condition conformes à cette norme. Il est connu que, la variabilité hydrologique qui résulte de la répartition saisonnière des pluies ou la variabilité interannuelle des précipitations ont des conséquences importantes sur la biologie et la dynamique des peuplements de poissons (Lévêque, 2017). D’après Albaret (1999), les alternances périodiques crue-étiage ont un impact majeur sur la biologie, la physiologie et l'écologie les populations des poissons. En outre, plusieurs facteurs peuvent influencer le coefficient de condition, dont la taille des gonades, le sexe, le contenu stomacal et la disponibilité d'aliments (Hossain et al., 2006). Comme Lalèyè (2006), Muzzalifah et al. (2015), Lederou et al. (2016) et Ibala Zamba et al. (2020), ces paramètres n'ont pas été pris en compte dans le traitement de données.

les poissons de la lagune Mvassa en saison de pluie. Une étude complémentaire sera effectuée pour prendre en compte d'autres facteurs comme la reproduction et les habitudes alimentaires afin de mieux comprendre ce phénomène. Cette étude rapporte des informations relatives à la biologie des poissons 
rencontrés dans la lagune Mvassa, mais montre aussi l'influence de la saison sur les relations poids-longueur et les coefficients de condition. La RPLs et le coefficient de condition sont utilisés pour les calculs des statistiques biologiques des populations de poissons. Ces

\section{REMERCIEMENT}

Les auteurs remercient Martin Bassafoula, Daniel Bavedila, ainsi que toute l'équipe de l'Institut national de Recherche en Sciences

\section{BIBLIOGRAPHIE}

Albaret JJ : 1994. Les poissons : biologie et peuplements. In: Environnement et Ressources aquatiques de Côte d'Ivoire. Tome II - Les milieux lagunaires. (Durand J.R., Dufour P., Guiral D. \& S.G.F. Zabi, éds), Paris: ORSTOM, Pp. 239-279.

Baby F, Tharian J, Abraham KM, Ramprasanth MR, Ali A \& Ranghavan R : 2011. Length-weight relationship and condition factor of an endemic stone sucker, Garra gotyla Stenorbynchus (Jerdon, 1849) from two opposite flowing rivers in southern western ghats. Journal of threatened taxa 3(6): 1851-1855.

Bagenal TB \& Tesch AT: 1978. Conditions and growth patterns in fresh water habitats. Blackwell scientific publications, Oxford, 75-89.

Batiabo Mikembi AL, Ibala Zamba A, Mamonekene V, Dembe Louvinguila Tenda H, Poaty Ngot FH. \& Vouidibio $\mathrm{J}:$ 2019b. Relations longueurs-poids et coefficients de condition pour 13 especes de poissons de la rivière Dzoumouna, affluent du cours inferieur $\mathrm{du}$ fleuve Congo (République du Congo). Journal of Animal and Plant Sciences 39(1): 6384-6393.

Béné C: 2005. Contribution of Inland Fisheries to rural Livelihoods and food security in Africa: An overview. In : Thieme M. L., Abell R., Stiassny M. L. J., Lehner B., résultats constituent une base des données importante pour les gestionnaires de ces eaux afin d'établir les mesures de gestion durable. Quoi que petite par sa taille, cette lagune joue un rôle capital pour les populations riveraines, qui tirent leurs subsistances de ces eaux.

Exactes et Naturelles de Pointe-Noire et tous ceux qui ont participé de loin ou de près dans la récolte des données.

Teugels G. G., Dinerstein E., Kamdem Toham A., Burgress N. \& Olson D. Freshwater Ecoregions of Africa and Madagascar: A Conservation Assessment. WWF, Oislandpress: Pp 6-10.

Calander KD: 1969. Handbook of Freshwater Fishery Biology. The Iowa State Univ. Press, Ames, LA Lowa State 1: 752 pp.

Écoutin JM \& Albaret JJ : 2003. Relation longueur-poids pour 52 espèces de poissons des estuaires et lagunes de l'Afrique de l'Ouest. Cybium 27(1): 3-9.

Froese R: 2006. Cube law, condition factor and weight-length relationships: History, meta-analysis and recommendations. Journal of Applied Ichthyology 22: 241-253.

Froese R, Thorson JT \& Reyes RB: 2014. A bayesian approach for estimating length-weight Relationship in fishes. Journal of Applied Ichthyology 30: 78-85.

Hossain MY, Ahmed ZF, Leunda PM, Jasmine S, Oscoz J, Miranda R \& Ohtomi J : 2006. Conditions, length-weight and length-length relationships of the Asian striped catfish Mystus vittatus (Bloch, 1794) in the Mathabhanga river, southwestern Bangladesh. Journal of Applied Ichthyology 22: 304-307.

Hossain MY, Mosaddequr RMd, Fulanda B, Jewel MAS, Ahamed F \& Ohtomi J: 2012. Length-weight and length-length relationships of five threatened fish 196 species from the Jamuna (Brahmaputra 
River tributary) River, northern Bangladesh. Journal of Applied Ichthyology 28: 275-277

Ibala-Zamba A, Vreven E, Mamonekene V \& Snoeks J: 2019. Fish community assemblages in relation to environmental variables in the Lefini River, middle Congo River basin (Republic of Congo). Cybium 43(1): 83-95.

Ibala-Zamba A, Vreven E, Mamonekene V, Musschoot T, Poaty Ngot HF \& Snoeks J : 2019. Length-weight relationships of 37 freshwater fish species from the Lefini river, middle Congo River basin (Republic of the Congo). Journal of Appleid Ichthyology 00: 1-6.

Kareem OK, Olanrewaju AN \& Orisasona O (2015). Length-weight Relationship and Condition factor of Chrysichthys nigrodigitatus and Schilbe mystus in Erelu Lake, Oyo State, Nigeria. Journal of Fisheries and Livestock Production 3 (4): $1-4$

Konan SK., Kouakou BK, Ohou MJ, Konan FK \& Dongui KB: 2017. Variation saisonnière des paramètres abiotiques de la lagune Aghien (Côte d'Ivoire). Journal of Applied Biosciences 120: 12042-12052.

Lalèyè PA: 2006. Length-weight and lengthlength relationships of fishes from the Ouémé River in Bénin (West Africa). Journal of Applied Ichthyology 22 (4): 330-333.

Le Creon ED: 1951. The length-weight relationship and seasonal cycle in gonadal weight and condition in the perch (Perca fluviatilis). Journal of Animal Ecology 20: 201-219.

Lederoun D, Laleye P, Vreven E \& Vandewalle P: 2016. Length-weight and lengthweight relationships and condition factor for 30 actinopterygian fish from the mono basin (Benin and Togo, West Africa). Cybium 40 (4): 267-274.
Leveque C: 2017. Variability of Climate and Hydrobiological Systems. In: Paugy D., Leveque C., \& Otero O., (eds): The inland water fishes of Africa: Diversity, Ecology and Human use, Institut de recherche pour le développement (IRD), Royal Museum for Central Africa (RMCA), Pp. 33-50.

Leveque C, Paugy D \& Teugels GG: 1990 (eds). Faune des poissons d'eaux douces et saumâtres de l'Afrique de l'Ouest. Volume 1, MRAC (Tervuren), ORSTOM (Paris), collection faune tropicale, $28: 384 \mathrm{pp}$.

Leveque C, Paugy D \& Teugels GG: 1992 (eds). Faune des poissons d'eaux douces et saumâtres de l'Afrique de l'Ouest. Volume 2, MRAC (Tervuren), ORSTOM (Paris), collection faune tropicale 28: 389-902 pp.

Lizama MAP \& Ambrosia AM: 2002. Condition factor in nine species of fish of characidae family in the upper Parana River floodplain. Brazilian Journal of Biology 62 (1) : 113-124.

Mamonekene V \& Teugels GG : 1993. Faune des poissons d'eaux douces de la réserve de la biosphère de Dimonika (Mayombe, Congo). Ann. Mus. Roy. Afr. Centr. MARC (Tervuren) \& UNESCO $272: 126 \mathrm{pp}$.

Mostarih MMM, Madani FE, Yahya HSA, Hachemi OE, Abdellaoui S \& Chafi A : 2016. Evaluation physico-chimique de la qualité de l'eau de la lagune de Nador-Nord du Maroc oriental après l'ouverture de la nouvelle passe. Journal of Materials and Environmental Science 7(12): 4795-5809.

Palomares MLD, Entsua-Mensah M \& OseiAbunyewa A: 1966. Length-weight relationships of fishes from tributaries of the Volta River, Ghana: part 2 and conclusion. NAGA, ICLARM Quat., 19 (1): 45-47.

Paugy D \& Leveque C: 2017. Impacts of human activities. In: Paugy $\mathrm{D}$, Lévêque 
C. \& Otero O. (eds) : The inland water fishes of Africa: Diversity, Ecology and Human use, Institut de recherche pour le développement (IRD), Musée royal de l'Afrique centrale (MRAC), Pp. 459-478.

Petrakis O \& Leveque C : 2017. Impacts of human activities. In : Paugy D., Leveque C. \& Otero O., (eds) : The inland water fishes of Africa : Diversity, Ecology and Human use, Institut de recherche pour le développement (IRD), Royal Museum for Central Africa (RMCA), Pp. 459-478.

RIOB (Réseau International des Organismes de Bassin) : 2015. Manuel de la gestion intégrée des ressources en eau dans les bassins des fleuves et des lacs. $95 \mathrm{pp}$.

Romdhani A, Ktari MH \& Mahe K 2013. Length-weight relationship and condition factor of phycis (linnaeus, 1766) and phycis blennoides (Brünnich, 1768) Actinopterygii, Gadiform, Phycidae] in the gulf of tunis. Bulletin de l'Institut National des Sciences et Technologies de la Mer de Salammbô $40:$ 3-13.

Shingleton AW : 2010. Allometry : the study of biological Scaling. Nature Education Knowledge 3(10) : 2 .

Sokal R \& Rohlf F: 1987. Introduction to Biostatistics. Freeman, New York, USA.

Stiassny LJS, Teugels GG \& Hopkins CD : 2007a. Poissons d'eaux douces et saumâtres de basse guinée, Ouest de l'Afrique Centrale. Collection faune et flore tropicales. 42, IRD, Volume 1: 800 pp.

Stiassny LJS, Teugels GG. \& Hopkins CD : 2007b. Poissons d'eaux douces et saumâtres de basse guinée, Ouest de l'Afrique Centrale. Collection faune et flore tropicales. 42, IRD, Volume 2: 603 pp.

Stiassny LJS : 1996. An overview of freshwater biodiversity: with some lessons from African fishes. Fisheries 21: 7-13.
Tah L., Bi Goore G. \& Da Costa KS : 2012. Length-weight relationships for 36 freshwater fish species from two tropical reservoirs: Ayamé I. \& Buyo, côte d'Ivoire. Revista de biologia tropical 60 (4) : 1847-1856.

Tomanova S: 2010. Relations longueur-poids pour les poissons d'eau douce en France. Rapport technique ONEMA, $13 \mathrm{pp}$.

Van Der Laan R., Fricke R. \& Eschmeyer WN : 2019. Eschmeyer's catalog of fishes: classification. (http://www.calacademy.org/scientists Lcatalog-of-fishes-classification/).

Zar J.H : 1999. Biostatistical Analysis. 4th Edition. Prentice-Hall, Englewood Cliffs, New Jersey, USA, 662 pp. 